Western University

Scholarship@Western

Aboriginal Policy Research Consortium International (APRCi)

7-2-2012

\title{
An intensive smoking intervention for pregnant Aboriginal and Torres Strait Islander women: a randomised controlled trial
}

Sandra J. Eade

Rob W. Sanson-Fisher

Mark Wenitong

Katie Panaretto

Catherine D'Este

See next page for additional authors

Follow this and additional works at: https://ir.lib.uwo.ca/aprci

Part of the Public Health Education and Promotion Commons

Citation of this paper:

Eade, Sandra J.; Sanson-Fisher, Rob W.; Wenitong, Mark; Panaretto, Katie; D'Este, Catherine; Gilligan, Conor; and Stewart, Jessica, "An intensive smoking intervention for pregnant Aboriginal and Torres Strait Islander women: a randomised controlled trial" (2012). Aboriginal Policy Research Consortium International (APRCi). 421.

https://ir.lib.uwo.ca/aprci/421 
Authors

Sandra J. Eade, Rob W. Sanson-Fisher, Mark Wenitong, Katie Panaretto, Catherine D'Este, Conor Gilligan, and Jessica Stewart 


\section{An intensive smoking intervention for pregnant Aboriginal and Torres Strait Islander women: a randomised controlled trial}

\section{Sandra J Eades BMed, PhD Head \\ Rob W Sanson-Fisher PhD, ClinMPsych, Laureate Professor of Health Behaviour ${ }^{2}$ \\ Mark Wenitong BMed \\ Senior Medical Officer \\ Katie Panaretto MB BS, \\ Population Healt Medical Officer \\ Catherine D'Este BMath PhD Professor of Biostatistics \\ Conor Gilligan BBiomedSc, PhD, Lecturer $^{2}$ \\ Jessica Stewart BA/LLB, MPP PhD Student \\ 1 Indigenous Maternal and Child Health Research Program, Baker IDI Heart and Diabetes Institute Melbourne, VIC. \\ 2 University of Newcastle Newcastle, NSW. \\ 3 Apunipima Cape York Health Council, Cairns, QLD. \\ 4 Queensland Aboriginal and Islander Health Council. Brisbane, QLD. \\ Sandra.eades@ bakeridi.edu.au}

MJA 2012; 197: 42-46 doi: 10.5694/mjall.10858

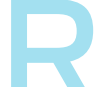

ates of smoking in pregnancy among Aboriginal and Torres Strait Islander women are high, ${ }^{1-3}$ with $52 \%$ smoking during pregnancy compared with $15 \%$ in non-Indigenous Australian women. ${ }^{3,4}$ Smoking during pregnancy among Aboriginal women is inversely related to socioeconomic status, ${ }^{4}$ and in qualitative studies, Aboriginal women have nominated stress as a major reason for smoking. ${ }^{5,6}$ Indigenous women in other developed countries also have higher rates of smoking during pregnancy than women in the general population. ${ }^{7-9}$

As smoking rates in the general population have fallen in highincome countries, smoking has become more closely related to entrenched social disadvantage. ${ }^{10} \mathrm{~A}$ review of interventions for promoting smoking cessation during pregnancy $^{10}$ showed that intervention was associated with a significant overall reduction in smoking during late pregnancy (risk ratio [RR], 0.94 [95\% CI, 0.93-0.96]). The review suggested there was a need for studies to refine interventions to address the specific needs of disadvantaged subpopulations. ${ }^{10}$

The aim of our study was to determine the effect of an intensive smoking cessation intervention for pregnant Aboriginal and Torres Strait Islander women on smoking rates at 36 weeks' gestation.

\section{Methods}

\section{Setting and participants}

Our study was conducted at three Aboriginal community-controlled health services located in urban communities in Queensland and Western Australia. Pregnant Aboriginal and Torres Strait Islander women who attended antenatal clinics at the participating health services were the target population.

Objective: To determine the effectiveness of an intensive quit-smoking intervention on smoking rates at 36 weeks' gestation among pregnant Aboriginal and Torres Strait Islander women.

Design: Randomised controlled trial.

Setting and participants: Pregnant Aboriginal and Torres Strait Islander women $(n=263)$ attending their first antenatal visit at one of three Aboriginal community-controlled health services between June 2005 and December 2009.

Intervention: A general practitioner and other health care workers delivered tailored advice and support to quit smoking to women at their first antenatal visit, using evidence-based communication skills and engaging the woman's partner and other adults in supporting the quit attempts. Nicotine replacement therapy was offered after two failed attempts to quit. The control ("usual care") group received advice to quit smoking and further support and advice by the GP at scheduled antenatal visits.

Main outcome measure: Self-reported smoking status (validated with a urine cotinine measurement) between 36 weeks' gestation and delivery.

Results: Participants in the intervention group $(n=148)$ and usual care group $(n=115)$ were similar in baseline characteristics, except that there were more women who had recently quit smoking in the intervention group than the control group. At 36 weeks, there was no significant difference between smoking rates in the intervention group (89\%) and the usual care group (95\%) (risk ratio for smoking in the intervention group relative to usual care group, $0.93[95 \% \mathrm{Cl}$, $0.86-1.08$ ]; $P=0.212$ ). Smoking rates in the two groups remained similar when baseline recent quitters were excluded from the analysis.

Conclusion: An intensive quit-smoking intervention was no more effective than usual care in assisting pregnant Aboriginal and Torres Strait Islander women to quit smoking during pregnancy. Contamination of the intervention across groups, or the nature of the intervention itself, may have contributed to this result.

Trial registration: Australian New Zealand Clinical Trials Registry ACTRN12609000929202.

Women were considered eligible to participate in the study if they were Aboriginal or Torres Strait Islanders; were attending their first antenatal appointment at one of the Aboriginal community-controlled health services at or before 20 weeks' gestation; were aged 16 years or older, were self-reported current smokers or recent quitters (quitting when they knew they were pregnant); and were residents of the local area. Recent quitters were included because there is evidence that such women often relapse, either later in pregnancy or after the birth. ${ }^{11,12}$ Women were excluded if their pregnancy was complicated by a mental illness or they were receiving treatment for chemical dependencies other than tobacco or alcohol use.
Participants were recruited between June 2005 and December 2008, with all final follow-up sessions (between 36 weeks' gestation and delivery) completed by December 2009. We were unable to report follow-up at 6 months postpartum owing to high rates of attrition.

Women who consented to participate completed a written questionnaire recording their social and demographic status and smoking history.

Smoking status was determined by asking women "Which of the following best describes your smoking status?", with possible responses being "regular smoker", "occasional smoker" or "quit since becoming pregnant". Women who smoked daily or occasionally were asked whether 
they had smoked in the past 7 days. Those who answered yes were defined as "current smokers". Women who self-reported quitting since becoming pregnant were defined as "recent quitters".

\section{Randomisation}

A randomised controlled design was used. To reduce the probability of contamination by providers delivering the intervention to both the intervention group and the usual care group, and to increase the ease with which health care providers could follow the experimental protocol, randomisation was by week of clinic attendance. An Excel computer program was used to randomly allocate weeks to intervention or control for all clinics. At the beginning of each week, clinics were notified of the allocation sequence and the research assistant flagged the clinical record of study participants to indicate whether they were allocated to the usual care or intervention group. The person generating the allocation sequence was not involved in participant recruitment. Participants were informed of the purpose of the study at recruitment but not of their allocated treatment group until after they had provided consent.

\section{Study intervention and outcome measures}

\section{Intervention development and principles}

The intervention was developed after a critical review of the literature and consultation with general practitioners, health care workers and community representatives in the health services.

Principles of the intervention were as follows: (i) it should be culturally sensitive and specific; (ii) the GP would advise the women to quit smoking by going "cold turkey"; (iii) the GP would ask women attempting to quit to voluntarily leave their cigarettes at the clinic before they left; (iv) significant others (including the woman's partner or other adult residents of the home) would be invited verbally and by mail to support the woman by attempting to quit smoking themselves and by supporting the woman's attempts to quit; (v) health care workers would be encouraged to provide evidence-based communication, including getting written agreement by the mother to quit, providing written instructions about strategies to cope with cravings for a cigarette, repeating and summarising key components of verbal quitting advice, asking the woman to recall the verbal messages to the health care provider, and filling in key gaps in the woman's recall; (vi) booster support sessions would be scheduled to strengthen support for women with early nicotine withdrawal symptoms; (vii) women who relapsed into smoking would be asked to continue making attempts to quit while pregnant; and (viii) nicotine replacement therapy would be offered to women who had failed two attempts at quitting.

Women in the intervention group were reassured that their care would not alter as a consequence of smoking.

\section{Details of the intervention}

For women in the intervention group, the intervention commenced at the woman's first antenatal visit, with a scripted invitation from the doctor for her to quit smoking (details of script available from the authors on request). Women who agreed to stop smoking were then advised by the doctor to quit "cold turkey" and to return to the clinic staff again in 3-5 days, given the pernicious nature of smoking. The doctor also checked whether the woman had understood the advice, repeated any components not understood, and gave the woman an appointment reminder card for her wallet, a fridge magnet and a letter for other household members requesting they support the woman as she attempted to quit smoking. Women were asked to bring a partner or support person with them on their second visit. If women indicated at the first antenatal visit that they didn't want to stop smoking, the doctor replied "I understand that it is difficult to stop smoking. However, because it is important, I would like to talk with you again about it when you next visit for antenatal care. Would that be OK?" Women could then indicate if they didn't want to talk about smoking again at future antenatal visits.

Follow-up visits were scheduled at 3-5 days and 7-10 days after the first antenatal visit to provide further support at a time when women would be experiencing major nicotine withdrawal symptoms. For women who were still smoking at the 7-10-day visit, nicotine replacement therapy was discussed and commenced for those who had no contraindications and wished to try it.

The initial intervention agent was the treating GP, with follow-up conducted by female Aboriginal or Torres Strait Islander health workers and midwives. Training was provided on site at each clinic at baseline and throughout the intervention period. A behavioural scientist and an Aboriginal doctor with GP experience provided the training.

Intervention training involved video demonstration, role play and presentation of relevant research literature. A study manual and a one-page guide with scripted advice for each participant enrolment and intervention session were provided to assist in implementing the intervention.

\section{Adherence to experimental protocol}

To ensure adherence to the protocol during the study, a number of visits were made by the lead investigators, and intervention refresher training was provided.

Doctors and other health care providers were also asked to complete a checklist of the essential intervention components after seeing each woman in the intervention group. The health care providers self-reported their adherence to the protocol for a subgroup of 66 women in the intervention group.

\section{Usual care}

Women in the usual care group received general advice from their GP about quitting smoking, based on existing brief intervention guidelines. ${ }^{13}$

\section{Follow-up}

Women were asked to provide the contact details of three people who would be able to contact them. The women were given a written appointment date and time for their next clinic visit. They were also phoned and sent text messages by mobile phone to remind them of each appointment. 


\section{Flow diagram of randomisation and recruitment of trial participants}

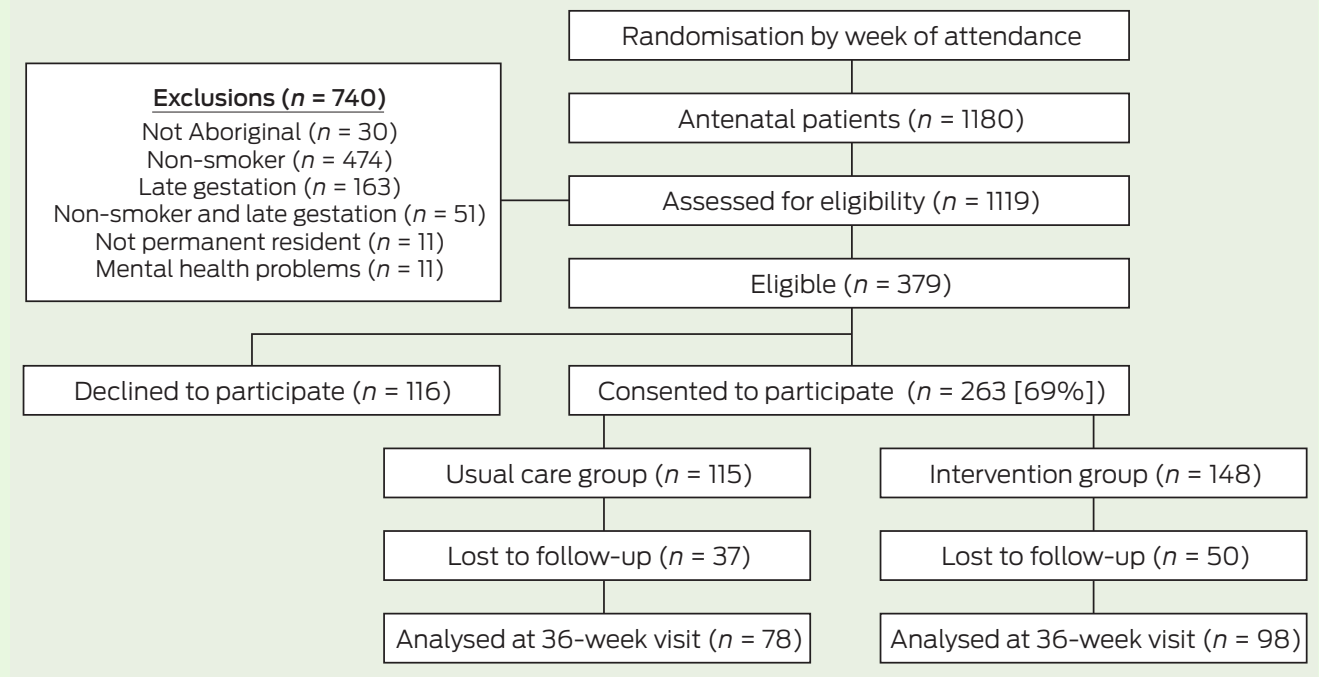

\section{Outcome measures}

Outcome assessments were conducted between 36 weeks' gestation and delivery. Women who reported they had quit smoking were asked to provide a urine sample for cotinine analysis, and a portion of the sample was frozen at $-20^{\circ} \mathrm{C}$ before analysis.

A Cozart microplate ELISA (enzyme-linked immunosorbent assay) test kit (Concateno, London, UK) was used for urine analysis. All samples were analysed in duplicate, with final results based on the average result of two paired wells. A woman was defined at follow-up as being a current smoker if (i) she reported that she had smoked in the previous 7 days; (ii) she reported that she had not smoked within the previous 7 days, but had a urine cotinine level of $\geqslant 250 \mathrm{ng} / \mathrm{mL}$ at the time; or (iii) she did not provide a urine sample. ${ }^{5}$

\section{Statistical methods}

\section{Statistical analysis}

Baseline characteristics of the two intervention groups are presented as frequency distributions for categorical variables and medians and quartiles for continuous variables. Because of the small numbers of quitters, exact logistic regression analyses were used to compare 36-week smoking status between the two groups after adjusting for recruitment centre. Relative risks and 95\% confidence intervals were estimated using the MantelHaenszel technique, with stratification by health service.
The primary analysis included women for whom follow-up data were available. Sensitivity analysis involved an intention-to-treat analysis, with women who were lost to follow-up considered as continuing smokers. Analyses were conducted using Stata statistical software, version 10.1 (StataCorp, College Station, Texas, USA).

\section{Sample size justification}

We planned to recruit 270 smokers into the study over a 30-month period (135 women in each of the intervention and control groups). We assumed that $80 \%$ of women $(108$ per group) could be followed up. This sample size for outcomes would allow detection of a $20 \%$ difference between groups in smoking cessation (assuming a quit rate of $15 \%$ in the control group), with $5 \%$ significance level and $80 \%$ power, and allowing for a design effect of 1.5 for clustering of participants within clinics.

\section{Consent and ethics approval}

Eligible women were informed about the study and invited to participate. Non-consenters were asked their age, marital status, number of children, address and whether they smoked.

Our study was approved by the University of Newcastle's Human Research Ethics Committee and the Western Australian Aboriginal Health Information and Ethics Committee.

\section{Results}

\section{Flow of participants}

A flow diagram of randomisation and recruitment of trial participants is shown in Box 1. Of the 263 women who consented to participate, 148 were in the intervention group and 115 in the usual care group. Twothirds of the women (176 [67\%]) completed the outcome assessment: 98 $(66 \%)$ in the intervention group and $78(68 \%)$ in the usual care group.

\begin{tabular}{|c|c|c|}
\hline Characteristic & $\begin{array}{l}\text { Usual care group } \\
\qquad(n=115)\end{array}$ & $\begin{array}{l}\text { Intervention group } \\
\quad(n=148)\end{array}$ \\
\hline $\begin{array}{l}\text { Median number of cigarettes smoked per day } \\
\text { (interquartile range) }\end{array}$ & $10.0(4.0-15.0)$ & $10.0(5.0-15.0)$ \\
\hline $\begin{array}{l}\text { Median number of weeks' gestation at } \\
\text { recruitment (interquartile range) }\end{array}$ & $12.0(8.0-19.0)$ & $12.0(8.0-17.0)$ \\
\hline Has a partner & $86(92 \%)$ & $118(88 \%)$ \\
\hline \multicolumn{3}{|l|}{ Smoking status } \\
\hline Regular smoker & $73(77 \%)$ & $92(67 \%)$ \\
\hline Occasional smoker & $14(15 \%)$ & $21(15 \%)$ \\
\hline Quit since becoming pregnant & $8(8 \%)$ & $24(18 \%)$ \\
\hline \multicolumn{3}{|l|}{ Location } \\
\hline Clinic 1 & $60(52 \%)$ & 86 (58\%) \\
\hline Clinic 2 & $39(34 \%)$ & $44(30 \%)$ \\
\hline Clinic 3 & $16(14 \%)$ & $18(12 \%)$ \\
\hline \multicolumn{3}{|l|}{ Parity } \\
\hline 0 & $28(30 \%)$ & $41(30 \%)$ \\
\hline 1 & $22(23 \%)$ & $30(22 \%)$ \\
\hline 2 or more & $44(47 \%)$ & $66(48 \%)$ \\
\hline
\end{tabular}




\begin{tabular}{|c|c|c|}
\hline Protocol domain & Protocol component & $\begin{array}{l}\text { Number (\%) } \\
\text { of women who } \\
\text { received component }\end{array}$ \\
\hline \multirow[t]{4}{*}{ Doctor's compliance at first antenatal visit } & Written agreement between woman and doctor to quit & $47(71 \%)$ \\
\hline & Advice that best way to quit was to go "cold turkey" & $49(74 \%)$ \\
\hline & Agreement to talk again & $42(64 \%)$ \\
\hline & Letter sent to support person & $38(58 \%)$ \\
\hline \multirow[t]{7}{*}{ Health worker's or nurse's compliance at first antenatal visit } & Advice about adverse effects of smoking & $16(24 \%)$ \\
\hline & Advice about avoiding triggers for cravings & $14(21 \%)$ \\
\hline & Advice about ways to cope with cravings & $15(23 \%)$ \\
\hline & Advice about benefits of quitting smoking & $15(23 \%)$ \\
\hline & Advice about multiple attempts needed to quit & $11(17 \%)$ \\
\hline & Advice about trying again even if relapse occurs & $10(15 \%)$ \\
\hline & Education pamphlets given & $14(21 \%)$ \\
\hline \multirow[t]{2}{*}{ Participant's compliance with follow-up visits } & Attended follow-up within 3-7 days & $42(64 \%)$ \\
\hline & Attended second follow-up at 7-10 days & $23(35 \%)$ \\
\hline Doctor prescribed NRT at third (7-10-day) visit & Prescribed NRT at third visit & $21(32 \%)$ \\
\hline
\end{tabular}

NRT = nicotine replacement therapy. $*$ Expressed as number (\%) of a subgroup of 66 women in the intervention group who received specific components of the structured behavioural change intervention.

\section{Baseline characteristics}

Women in both groups were similar in terms of the median number of cigarettes smoked per day, number of weeks' gestation at recruitment, and parity (Box 2). Compared with the usual care group, a slightly higher proportion of women in the intervention group were from Clinic 1, and a slightly lower proportion had a partner. The intervention group had a higher proportion of recent quitters than the control group. Consequently, we undertook a post-hoc subgroup analysis that included only those women classified as regular or occasional smokers at baseline.

\section{Provider adherence to protocol}

In over $64 \%$ of intervention consultations, doctors adhered to the protocol in providing key components of the intervention. Lower proportions of nurses and health workers recorded that they had provided intervention advice (Box 3).

\section{Trial outcomes}

At 36-week follow-up, there was no significant difference in smoking rates between the two groups. Of the women followed up, $87(89 \%)$ in the intervention group and 72 (95\%) in the usual care group were smokers (RR for intervention versus usual care, 0.93 [95\% CI, 0.86-1.08]; $P=0.212$ ) (Box 4).

Smoking status at follow-up was not available for two women in the usual care group. Cotinine measures for 16 of 34 women who self-reported that they had quit were missing (seven from the intervention group and nine from the usual care group), and a further three had cotinine levels $\geqslant 250 \mathrm{ng} / \mathrm{mL}$. These 19 women were classified as continuing smokers.

As expected, smoking rates were higher in the intention-to-treat analysis, with 137 smokers (93\%) in the intervention group and 111 smokers $(97 \%)$ in the usual care group (RR, 0.95 [95\% CI, 0.90-1.01]; $P=0.207$ ) (Box 4).

Subgroup analysis excluding baseline recent quitters showed no significant differences in smoking rates between the intervention group and the usual care group $(P=0.992)$ (Box $5)$. Corresponding figures for the intention-to-treat analysis were 123 smokers (99\%) in the intervention group and 105 smokers (98\%) in the usual care group (RR, 1.01 [95\% CI, 0.98-1.04]; $P=0.965)$ (Box 5).

\section{Discussion}

At 36-week follow-up, we found no significant difference in smoking rates between pregnant women who received a high-intensity quit-smoking intervention compared with those who received usual care. To our knowledge, ours are the first cotininevalidated quit rates reported for Aboriginal and Torres Strait Islander women in any intervention study.

Over $70 \%$ of women in the intervention group agreed to try to quit smoking when asked by the GP at their first antenatal visit, and over $60 \%$ attended a visit scheduled within 3-7 days of agreeing to make this quit attempt. These findings indicate a high degree of willingness among pregnant Aboriginal and Torres Strait Islander women who smoke to engage with quit attempts.

\begin{tabular}{|c|c|c|c|c|}
\hline & Usual care group & Intervention group & $P^{\dagger}$ & $\operatorname{RR}(95 \% \mathrm{Cl})^{\ddagger}$ \\
\hline Analysis of women with complete follow-up data & $72 / 76(95 \%)^{5}$ & $87 / 98(89 \%)$ & 0.212 & $0.93(0.86-1.08)$ \\
\hline Intention-to-treat analysis & $111 / 115$ (97\%) & $137 / 148(93 \%)$ & 0.207 & $0.95(0.90-1.01)$ \\
\hline
\end{tabular}

$\mathrm{RR}=$ risk ratio. $*$ Women with a missing cotinine sample or with cotinine level $\geqslant 250 \mathrm{ng} / \mathrm{mL}$ were classified as smokers. $\dagger P$ value for testing difference in quit rates was calculated using exact logistic regression. $P$ was adjusted for centre. $¥ R R(95 \% \mathrm{Cl})$ was estimated using the Mantel-Haenszel technique. $\S$ Excludes two women with missing smoking status. 9 Women lost to follow-up or with missing smoking status were classified as current smokers. 
5 Number (\%) of women smoking at $\geqslant 36$ weeks' gestation, based on cotinine validation of self-report:* complete outcome versus intention-to-treat analysis, excluding baseline recent quitters

\begin{tabular}{|c|c|c|c|c|}
\hline & Usual care group & Intervention group & $P^{\dagger}$ & $\operatorname{RR}(95 \% \mathrm{Cl})^{\ddagger}$ \\
\hline $\begin{array}{l}\text { Analysis of women with complete follow-up data } \\
\text { (excluding baseline recent quitters) }\end{array}$ & $68 / 70(97 \%)^{\varsigma}$ & $80 / 81(99 \%)$ & 0.992 & $1.01(0.96-1.06)$ \\
\hline Intention-to-treat analysis ${ }^{\natural}$ & 105/107 (98\%) & $123 / 124(99 \%)$ & 0.965 & $1.01(0.98-1.04)$ \\
\hline
\end{tabular}

In our study design we estimated that women who received the intervention would have a $20 \%$ higher absolute quit rate than women in the control group. We based this estimate on the high proportion of Aboriginal and Torres Strait Islander women who smoked during pregnancy compared with non-Indigenous pregnant women. We expected to find that a large number of women would have lower levels of nicotine dependence and would be able to quit with intensive support. A previous study of Aboriginal people had shown high levels of motivation to quit among pregnant women compared with other adults. ${ }^{14}$ However, our expectations were not borne out by our results.

At baseline, there were more recent quitters in the intervention group than the control group. Post-hoc analysis of the subgroup of women that excluded baseline quitters showed that smoking rates in the intervention and usual care groups were more similar at follow-up than at baseline. This suggests that most of the non-smokers at follow-up were those who were recent quitters at baseline. One possible reason for this is that, in this population, once women quit smoking in pregnancy they tend to remain quitters for the duration of the pregnancy. This is contrary to previous evidence that women who quit smoking when they become pregnant are likely to relapse later in the pregnancy. ${ }^{11,12}$

Our study had a number of positive aspects. The brief intervention was innovative and provided a fresh focus on smoking reduction for these services, which are accustomed to struggling with large numbers of tobacco users.

Methodological limitations of our study were the high (over 30\%) loss to follow-up, lack of allocation concealment, and the potential for contamination between groups (see below).
Despite extensive efforts, we were unable to limit the loss to follow-up

High staff turnover increased the difficulty of ensuring adherence to the intervention protocol. Extensive retraining was required during the study. There was potential for contamination by the providers delivering the intervention to both the intervention and usual care women. Although we randomised by week of clinical attendance to reduce the possibility that providers would contaminate groups at the initial visit, providers were required to change their consultation approach after recruitment according to each woman's study allocation. Our decision to randomise by week of attendance resulted in a lack of allocation concealment among the people recruiting women to the study. The fact that more women were recruited to the intervention group than the usual care group may be a result of the lack of allocation concealment.

Our study has implications for intervention research and efforts to reduce smoking among Aboriginal people. Studies other than placebocontrolled drug trials that randomly allocate individual participants to receive interventions are likely to be contaminated. Future studies should consider cluster randomised designs.

Our study highlights some of the difficulties of undertaking research in this field. However, given the serious impact of smoking in this group, continuing research and work in this area is an urgent priority.

Acknowledgements: We wish to thank the pregnant Indigenous women who participated in our study and the collaborating Aboriginal health services (Wuchopperen Health Service, Cairns; Townsville Aboriginal and Islander Health Service, Townsville; and Derbarl Yerrigan Health Service, Perth). We also wish to acknowledge our project workers at each site: Nancy Stephens, Jasmin CockatooCollins, Kaye Thompson, Lynette Anderson, Rozlyn Yarran and Joan Fraser. Our study was funded by National Health and Medical Research Council project grants 320851 and 510771 and program grant 472600 .

Competing interests: No relevant disclosures.
Received 8 Jul 2011, accepted 14 Mar 2012.

1 de Costa C, Child A. Pregnancy outcomes in urban Aboriginal women. Med J Aust 1996; 164: 523-526.

2 Eades SJ, Read AW. Infant care practices in a metropolitan Aboriginal population. J Paediatr Child Health 1999; 35: 541-544.

3 Laws P, Sullivan EA. Australia's mothers and babies 2007. Canberra: Australian Institute of Health and Welfare, 2009. (AIHW Cat. No. PER 48; Perinatal Statistics Series No. 23.) http:// www.aihw.gov.au/publication-detail/?id= 6442468312 (accessed Apr 2012).

4 Thrift AP, Nancarrow H, Bauman AE. Maternal smoking during pregnancy among Aboriginal women in New South Wales is linked to social gradient. Aust N Z J Public Health 2011; 35: 337-342.

5 Gilligan C, Sanson-Fisher R, Eades S, et al. Assessing the accuracy of self-reported smoking status and impact of passive smoke exposure among pregnant Aboriginal and Torres Strait Islander women using cotinine biochemical validation. Drug Alcohol Rev 2010; 29: 35-40.

6 Wood L, France K, Hunt K, et al. Indigenous women and smoking in pregnancy: knowledge, cultural contexts and barriers to cessation. Soc Sci Med 2008; 66: 2378-2389.

7 Tipene-Leach D, Hutchison L, Tangiora A, et al. SIDS-related knowledge and infant care practices among Maori mothers. NZ Med J 2010; 123: 88-96.

8 Centers for Disease Control and Prevention (CDC). Maternal, pregnancy, and birth characteristics of Asians and native Hawaiians/ Pacific Islanders - King County, Washington, 2003-2008. MMWR Morb Mortal Wkly Rep 2011; 60: $211-213$.

9 lyasu S, Randall LL, Welty TK, et al. Risk factors for sudden infant death syndrome among northern plains Indians. JAMA 2002; 288: 2717-2723.

10 Lumley J, Chamberlain C, Dowswell T, et al. Interventions for promoting smoking cessation during pregnancy. Cochrane Database Syst Rev 2009; (3): CD001055.

11 Eades S. Bibbulung Gnarneep (Solid Kid): a longitudinal study of a population based cohort of urban Aboriginal children in Western Australia: determinants of health outcomes during early childhood of Aboriginal children residing in an urban area [PhD thesis]. Perth: University of Western Australia, 2003

12 Najman JM, Lanyon A, Anderson M, et al. Socioeconomic status and maternal cigarette smoking before, during and after a pregnancy. Aust N Z J Public Health 1998; 22: 60-66.

13 Queensland Health. Smoke check - Indigenous smoking program. http://www.health.qld.gov. au/atod/prevention/smokecheck.asp (accessed May 2012).

14 Harvey D, Tsey K, Cadet-James Y, et al. An evaluation of tobacco brief interventions training in three indigenous health care settings in north Queensland. Aust N Z J Public Health 2002; 26: 426-431. 